PROCEEDINGS OF THE

AMERICAN MATHEMATICAL SOCIETY

Volume 134, Number 2, Pages 605-615

S 0002-9939(05)07997-9

Article electronically published on June 29, 2005

\title{
HEREDITARY TOPOLOGICAL DIAGONALIZATIONS AND THE MENGER-HUREWICZ CONJECTURES
}

\author{
TOMEK BARTOSZYŃSKI AND BOAZ TSABAN
}

(Communicated by Alan Dow)

\begin{abstract}
We consider the question of which of the major classes defined by topological diagonalizations of open or Borel covers is hereditary. Many of the classes in the open case are not hereditary already in ZFC, and none of them are provably hereditary. This is in contrast with the Borel case, where some of the classes are provably hereditary. Two of the examples are counter-examples of sizes $\mathfrak{d}$ and $\mathfrak{b}$, respectively, to the Menger and Hurewicz Conjectures, and one of them answers a question of Steprans on perfectly meager sets.
\end{abstract}

\section{INTRODUCTION}

1.1. Selection principles. Let $\mathscr{A}$ and $\mathscr{B}$ be collections of covers of a topological space $X$. The following selection hypotheses have a long history in the case where the collections $\mathscr{A}$ and $\mathscr{B}$ are topologically significant.

$\mathrm{S}_{1}(\mathscr{A}, \mathscr{B})$ : For each sequence $\left\{\mathcal{U}_{n}\right\}_{n \in \mathbb{N}}$ of members of $\mathscr{A}$, there exist members $U_{n} \in \mathcal{U}_{n}, n \in \mathbb{N}$, such that $\left\{U_{n}\right\}_{n \in \mathbb{N}} \in \mathscr{B}$.

$\mathrm{S}_{\text {fin }}(\mathscr{A}, \mathscr{B})$ : For each sequence $\left\{\mathcal{U}_{n}\right\}_{n \in \mathbb{N}}$ of members of $\mathscr{A}$, there exist finite (possibly empty) subsets $\mathcal{F}_{n} \subseteq \mathcal{U}_{n}, n \in \mathbb{N}$, such that $\bigcup_{n \in \mathbb{N}} \mathcal{F}_{n} \in \mathscr{B}$.

$\mathrm{U}_{\text {fin }}(\mathscr{A}, \mathscr{B})$ : For each sequence $\left\{\mathcal{U}_{n}\right\}_{n \in \mathbb{N}}$ of members of $\mathscr{A}$ which do not contain a finite subcover, there exist finite (possibly empty) subsets $\mathcal{F}_{n} \subseteq \mathcal{U}_{n}$, $n \in \mathbb{N}$, such that $\left\{\cup \mathcal{F}_{n}\right\}_{n \in \mathbb{N}} \in \mathscr{B}$.

1.2. Special covers. Let $X$ be a set of reals. In the following definitions, we always require that $X$ is not contained in any member of the cover. An $\omega$-cover $\mathcal{U}$ of $X$ is a cover of $X$ such that each finite subset of $X$ is contained in some member of $\mathcal{U} . \mathcal{U}$ is a $\tau$-cover if it is large (that is, each member of $X$ is contained in infinitely many members of the cover), and for each $x, y \in X$, (at least) one of the sets $\{U \in \mathcal{U}: x \in U, y \notin U\}$ and $\{U \in \mathcal{U}: y \in U, x \notin U\}$ is finite. $\mathcal{U}$ is a $\gamma$-cover if it is infinite, and each element of $X$ belongs to all but finitely many members of the cover. Let $\mathcal{O}, \Omega, \mathrm{T}$, and $\Gamma$ denote the collections of countable open covers, $\omega$-covers, $\tau$-covers, and $\gamma$-covers of $X$, respectively, and let $\mathcal{B}, \mathcal{B}_{\Omega}, \mathcal{B}_{\mathrm{T}}, \mathcal{B}_{\Gamma}$ be the corresponding countable Borel covers. The diagonalization properties of these types of covers were extensively studied in, e.g., [18, 11, 20, 24. Many of these

Received by the editors January 4, 2004 and, in revised form, September 20, 2004.

2000 Mathematics Subject Classification. Primary 54G20, 54G15, 54D20.

Key words and phrases. Selection principles, strong $\gamma$-set, Menger property, Hurewicz property. The first author was partially supported by NSF grant DMS 0200671.

This paper constitutes a part of the second author's doctoral dissertation at Bar-Ilan University. 
properties turn out to be equivalent [11, 24]; the classes which survived thus far appear in Figure 1 (for the open case). Some of the classes which are distinct in the open case coincide in the Borel case [20].

In the diagram, each property $P$ appears together with its critical cardinality non $(P)$, that is, the minimal size of a set of reals which does not satisfy that property. (See 6, 4] for the definitions of these and other constants mentioned in the paper.) The arrows in this diagram denote inclusion.

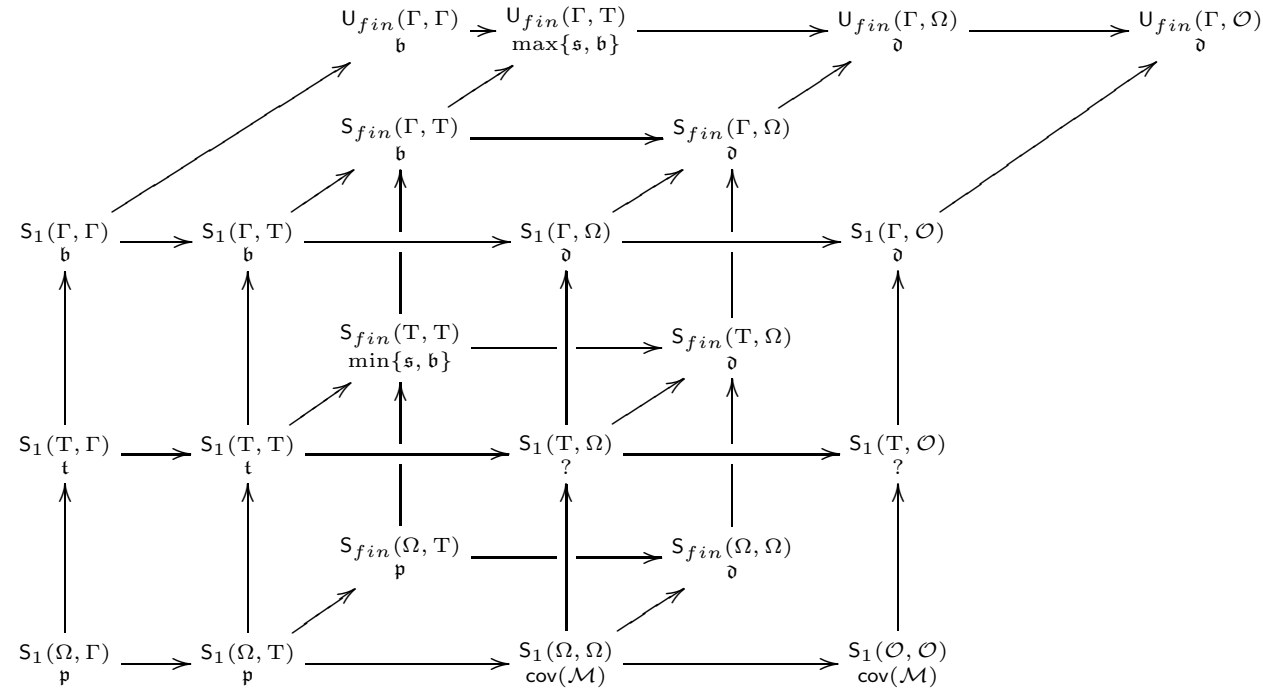

FiguRE 1. The surviving classes for the open case

1.3. Hereditary properties. If $\mathscr{A}$ and $\mathscr{B}$ are collections of open covers and $\Pi \in$ $\left\{\mathrm{S}_{1}, \mathrm{~S}_{\text {fin }}, \mathrm{U}_{\text {fin }}\right\}$, then $\Pi(\mathscr{A}, \mathscr{B})$ is closed under taking closed subsets [1]. Similarly, if $\mathscr{A}$ and $\mathscr{B}$ are collections of Borel covers and $\Pi \in\left\{\mathrm{S}_{1}, \mathrm{~S}_{\text {fin }}, \mathrm{U}_{\text {fin }}\right\}$, then $\Pi(\mathscr{A}, \mathscr{B})$ is closed under taking Borel subsets [20. We say that a class (or a property) is hereditary if it is closed under taking arbitrary subsets. A natural question which rises is: Which of these classes is provably hereditary? We show that some of the classes in the Borel case, but none of the classes in the open case, are provably hereditary.

For some of the classes in the open case, no additional axioms beyond ZFC are required in order to disprove their being hereditary. We describe two nontrivial examples which are in particular counter-examples to conjectures of Menger and Hurewicz.

\section{The Borel CASE}

Proposition 1. $\mathrm{S}_{1}(\mathcal{B}, \mathcal{B})$ is hereditary.

Proof. Assume that $X$ satisfies $\mathrm{S}_{1}(\mathcal{B}, \mathcal{B})$ and that $Y$ is a subset of $X$. Assume that $\left\{\mathcal{U}_{n}\right\}_{n \in \mathbb{N}}$ is a sequence of countable Borel covers of $Y$.

For each $n$, define $\mathcal{V}_{n}=\mathcal{U}_{n} \cup\left\{X \backslash \cup \mathcal{U}_{n}\right\}$. Then each $\mathcal{V}_{n}$ is a countable Borel cover of $X$, and we can choose for each $n$ an element $V_{n} \in \mathcal{V}_{n}$ such that $\left\{V_{n}\right\}_{n \in \mathbb{N}}$ 
is a cover of $X$. Define $U_{n}=V_{n}$ if $V_{n} \neq X \backslash \cup \mathcal{U}_{n}$, otherwise let $U_{n}$ be an arbitrary element of $\mathcal{U}_{n}$. Then $\left\{U_{n}\right\}_{n \in \mathbb{N}}$ is a cover of $Y$ and for each $n, U_{n} \in \mathcal{U}_{n}$.

All classes in the Borel case of the top plane of Figure 1 are hereditary.

Proposition 2. For each $\Pi \in\left\{\mathrm{S}_{1}, \mathrm{~S}_{\text {fin }}, \mathrm{U}_{\text {fin }}\right\}$ and each $\mathscr{B} \in\left\{\mathcal{B}, \mathcal{B}_{\Omega}, \mathcal{B}_{\mathrm{T}}, \mathcal{B}_{\Gamma}\right\}$, $\Pi\left(\mathcal{B}_{\Gamma}, \mathscr{B}\right)$ is hereditary.

Proof. The proof for this is similar to that of Proposition 1 If $\mathcal{U}=\left\{U_{n}\right\}_{n \in \mathbb{N}}$ is a countable Borel $\gamma$-cover of a subset $Y$ of $X$, then

$$
B_{\mathcal{U}}=\left\{x \in X \text { : for infinitely many } n, x \notin U_{n}\right\}
$$

is a Borel subset of $X$ disjoint from $Y$. We claim that $\mathcal{V}=\left\{U_{n} \cup B_{\mathcal{U}}\right\}_{n \in \mathbb{N}}$ is a (countable Borel) $\gamma$-cover of $X$. It is easy to see that each $x \in X$ is contained in all but finitely many members of $\mathcal{V}$. It remains to show that the cover is infinite. As $Y$ is not contained in any element of $\mathcal{U}$ and $B_{\mathcal{U}}$ is disjoint from $Y, X$ is not contained in any member of $\mathcal{V}$. Moreover, each finite subset of $X$ is contained in some element of $\mathcal{V}$. Thus, $\mathcal{V}$ is an $\omega$-cover of $X$, and in particular it is infinite.

Problem 3. Which of the remaining classes (which involve Borel covers) are provably hereditary?

Recently, Miller answered a part of this problem by showing that no class between $\mathrm{S}_{1}\left(\mathcal{B}_{\Omega}, \mathcal{B}_{\Gamma}\right)$ and $\mathrm{S}_{f i n}(\Omega, \mathrm{T})$ (inclusive) is provably hereditary [15].

Consistency facts. Borel's Conjecture, which was proved consistent by Laver, implies that the class $S_{1}(\mathcal{O}, \mathcal{O})$ (and the classes below it) reduces to contain only the countable sets of reals. Thus, it is consistent that all classes below $\mathrm{S}_{1}(\mathcal{O}, \mathcal{O})$ are hereditary.

A set of reals $X$ is a $\sigma$-set if each $G_{\delta}$ set in $X$ is also an $F_{\sigma}$ set in $X$. By 20], every element of $\mathrm{S}_{1}\left(\mathcal{B}_{\Gamma}, \mathcal{B}_{\Gamma}\right)$ is a $\sigma$-set. It is consistent that every $\sigma$-set of real numbers is countable [14. Consequently, it is consistent that all classes below $\mathrm{S}_{1}\left(\mathcal{B}_{\Gamma}, \mathcal{B}_{\Gamma}\right)$ are hereditary.

It is a major open problem whether it is consistent that every uncountable set of real numbers can be mapped onto a dominating subset of ${ }^{\mathbb{N}} \mathbb{N}$ by a Borel function. Such a consistency result would imply the consistency of all classes considered in this paper for the Borel case being hereditary.

\section{THE OPEN CASE}

A quasiordering $\leq^{*}$ is defined on the Baire space ${ }^{\mathbb{N}} \mathbb{N}: f \leq^{*} g$ if $f(n) \leq g(n)$ for all but finitely many $n$. A subset $Y$ of ${ }^{\mathbb{N}} \mathbb{N}$ is dominating if for each $g$ in ${ }^{\mathbb{N}} \mathbb{N}$ there exists $f \in Y$ such that $g \leq^{*} f$. It is bounded if there exists $g \in{ }^{\mathbb{N}}$ such that for each $f \in Y, f \leq^{*} g$. Cantor's space ${ }^{\mathbb{N}}\{0,1\}$ of infinite binary sequences is equipped with the product topology. Identify ${ }^{\mathbb{N}}\{0,1\}$ with $P(\mathbb{N})$ by characteristic functions. Also, denote by $[\mathbb{N}]^{\aleph_{0}}$ (respectively, $\left.[\mathbb{N}]^{<\aleph_{0}}\right)$ the collection of all infinite (respectively, finite) sets of natural numbers.

Assuming Martin's Axiom (or just $\mathfrak{p}=\mathfrak{c}$ ), there exists $X \subseteq P(\mathbb{N})$ of size $\mathfrak{c}$ such that $X$ satisfies $\mathrm{S}_{1}(\Omega, \Gamma)$ but $X \backslash[\mathbb{N}]^{<\aleph_{0}}$ does not satisfy $\mathrm{S}_{1}(\Omega, \Gamma)[8$. We will modify the construction of $[8$, to get a stronger result. A space $X$ is a $\tau$-set $[22$ if each clopen $\tau$-cover of $X$ contains a $\gamma$-cover of $X$. 
Theorem 4. Assume that $\mathfrak{p}=\mathfrak{c}$. Then there exists $X \subseteq P(\mathbb{N})$ such that $X$ satisfies $\mathrm{S}_{1}(\Omega, \Gamma)$ but $X \backslash[\mathbb{N}]^{<\aleph_{0}}$ does not satisfy $\mathrm{U}_{\text {fin }}(\Gamma, \mathcal{O})$. (Moreover, $X$ is a $\tau$-set and $X \backslash[\mathbb{N}]^{<\aleph_{0}}$ is not a $\tau$-set.)

Proof. For $y \subseteq \mathbb{N}$, define $y^{*}=\left\{x \subseteq \mathbb{N}: x \subseteq^{*} y\right\}$.

Lemma 5 ([8]). Assume that $\mathcal{G}$ is an open $\omega$-cover of $[\mathbb{N}]^{<\aleph_{0}}$. Then for each infinite $x \subseteq \mathbb{N}$ there exists an infinite $y \subseteq x$ such that $\mathcal{G}$ contains a $\gamma$-cover of $y^{*}$.

Identify $P(\mathbb{N})$ with the collection of strictly increasing functions in ${ }^{\mathbb{N}} \mathbb{N}$ by taking increasing enumerations. When $a \in[\mathbb{N}]^{\aleph_{0}}, \vec{a}$ denotes the increasing enumeration of $a$.

Let $\left\{\mathcal{G}_{\alpha}: \alpha<\mathfrak{c}\right\}$ enumerate all countable families of open sets in $P(\mathbb{N})$, let $\left\{\vec{d}_{\alpha}: \alpha<\mathfrak{c}\right\}$ be a dominating subset of ${ }^{\mathbb{N}} \mathbb{N}$, and let $\left\{a_{\alpha}: \alpha<\mathfrak{c}\right\} \subseteq[\mathbb{N}]^{\aleph_{0}}$ enumerate all infinite coinfinite subsets of $\mathbb{N}$. For convenience, for $x, y \in[\mathbb{N}]^{\aleph_{0}}$ we define

$$
\operatorname{sub}(x, y)= \begin{cases}x, & x \subseteq^{*} y, \\ x \backslash y, & \text { otherwise. }\end{cases}
$$

Thus $\operatorname{sub}(x, y) \in[\mathbb{N}]^{\aleph_{0}}, \operatorname{sub}(x, y) \subseteq x$, and either $\operatorname{sub}(x, y) \subseteq^{*} y$ or else $\operatorname{sub}(x, y) \subseteq^{*}$ $\mathbb{N} \backslash y$.

We construct by induction a dominating subset $\left\{\vec{x}_{\alpha}: \alpha<\mathfrak{c}\right\}$ of ${ }^{\mathbb{N}} \mathbb{N}$, such that $\left\{x_{\alpha}: \alpha<\mathfrak{c}\right\} \subseteq[\mathbb{N}]^{\aleph_{0}}$ is a (special type of a) tower, as follows.

For a limit $\alpha$, use $\alpha<\mathfrak{c}=\mathfrak{b}=\mathfrak{t}$ to get a pseudo intersection $p$ of $\left\{x_{\beta}: \beta<\alpha\right\}$ and a function $b \in{ }^{\mathbb{N}} \mathbb{N}$ which bounds $\left\{\vec{d}_{\beta}: \beta<\alpha\right\}$. Choose an infinite $q \subseteq p$ such that $\vec{b} \leq^{*} \vec{q}$, e.g., $\vec{q}(n)=\min \{k \in p: \vec{q}(n-1), \vec{b}(n)<k\}$. Then set $x_{\alpha}=\operatorname{sub}\left(q, a_{\alpha}\right)$.

The successors $x_{\alpha+1}$ are constructed as follows. If $\mathcal{G}_{\alpha}$ is not an $\omega$-cover of $X_{\alpha}=$ $\left\{x_{\beta}: \beta<\alpha\right\} \cup[\mathbb{N}]^{<\aleph_{0}}$, set $x_{\alpha+1}=\operatorname{sub}\left(x_{\alpha}, a_{\alpha+1}\right)$ (this case is not particularly interesting). Otherwise do the following: As $\left|X_{\alpha}\right|<\mathfrak{p}, \mathcal{G}_{\alpha}$ contains a $\gamma$-cover of $X_{\alpha}$. By Lemma 5, there exists an infinite $p \subseteq x_{\alpha}$ such that this $\gamma$-cover (which is in particular an $\omega$-cover of $[\mathbb{N}]^{<\aleph_{0}}$ ) contains a $\gamma$-cover $\left\{G_{n}\right\}_{n \in \mathbb{N}}$ of $p^{*}$. Observe that $\left\{G_{n}\right\}_{n \in \mathbb{N}}$ is a $\gamma$-cover of $\left\{x_{\beta}: \beta<\alpha\right\} \cup p^{*}$. Now, as in the first case, take an infinite $q \subseteq p$ such that $\vec{q}$ bounds $\left\{\vec{d}_{\beta}: \beta<\alpha+1\right\}$, and set $x_{\alpha+1}=\operatorname{sub}\left(q, a_{\alpha+1}\right)$.

Set $X=\left\{x_{\alpha}: \alpha<\mathfrak{c}\right\} \cup[\mathbb{N}]^{<\aleph_{0}}$. The properties follow, as in Theorems 2.14 and 3.7 of 22 . Briefly: To prove that $X$ satisfies $\mathrm{S}_{1}(\Omega, \Gamma)$, it suffices to show that each $\omega$-cover of $X$ contains a $\gamma$-cover of $X\left[9\right.$. By the construction, if $\mathcal{G}_{\alpha}$ is an $\omega$-cover of $X$, then it contains a $\gamma$-cover of $\left\{x_{\beta}: \beta \leq \alpha\right\} \cup x_{\alpha+1}^{*} \supseteq X$.

Each $\gamma$-set is a $\tau$-set. However, a tower is never a $\tau$-set [22]. $X \backslash[\mathbb{N}]<\aleph_{0}$ is a tower: Let $a \in[\mathbb{N}]^{\aleph_{0}}$. Take an infinite coinfinite $a_{\alpha} \subseteq a$. Then either $x_{\alpha} \subseteq^{*} a_{\alpha}$, or else $x_{\alpha} \subseteq^{*} \mathbb{N} \backslash a_{\alpha}$. Therefore, $a \nsubseteq^{*} x_{\alpha}$.

Finally, by a theorem of Hurewicz, a zero-dimensional space $X$ satisfies $\mathrm{U}_{\text {fin }}(\Gamma, \mathcal{O})$ if, and only if, for every continuous function $\Psi: X \rightarrow{ }^{\mathbb{N}} \mathbb{N}, \Psi[X]$ is not dominating. As $X \backslash[\mathbb{N}]^{<\aleph_{0}}$ is dominating, it does not satisfy $\bigcup_{f i n}(\Gamma, \mathcal{O})$.

A construction as in Theorem 4 cannot give information in the Borel cases: e.g., if $X$ satisfies $\mathrm{S}_{1}\left(\mathcal{B}_{\Omega}, \mathcal{B}_{\Gamma}\right)$ and $A$ is countable, then $X \backslash A$ is a Borel subset of $X$ and therefore satisfies $S_{1}\left(\mathcal{B}_{\Omega}, \mathcal{B}_{\Gamma}\right)$, too. However, this construction can be strengthened to obtain a strong $\gamma$-set [8]. A set $X$ of reals is a strong $\gamma$-set if, and only if, for each sequence $\left\{\mathcal{U}_{n}\right\}_{n \in \mathbb{N}}$, where for each $n \mathcal{U}_{n}$ is an $n$-cover of $X$, there exist elements $U_{n} \in \mathcal{U}_{n}, n \in \mathbb{N}$, such that $\left\{U_{n}\right\}_{n \in \mathbb{N}}$ is a $\gamma$-cover of $X[25$. 
Theorem 6. Assume Martin's Axiom. Then there exists $X \subseteq P(\mathbb{N})$ such that $X$ is a strong $\gamma$-set but $X \backslash[\mathbb{N}]^{<\aleph_{0}}$ does not satisfy $\bigcup_{\text {fin }}(\Gamma, \mathcal{O})$ and is not a $\tau$-set.

Proof. We carry out the same construction as in Theorem 4 but replace Lemma 5 with the following one, which is also due to Galvin and Miller [8].

Lemma 7 (MA). Assume that $X \subseteq P(\mathbb{N})$ is such that $|X|<\mathfrak{c}$, and $x \in[\mathbb{N}]^{\aleph_{0}}$. Then for each sequence $\left\{\mathcal{U}_{n}\right\}_{n \in \mathbb{N}}$ of open n-covers of $X \cup[\mathbb{N}]^{<\aleph_{0}}$ there exists an infinite subset $y$ of $x$ and a sequence $\left\{V_{n}\right\}_{n \in \mathbb{N}}$ such that for each $n, V_{n} \in \mathcal{U}_{n}$, and $\left\{V_{n}\right\}_{n \in \mathbb{N}}$ is a $\gamma$-cover of $X \cup y^{*}$.

This allows us to carry out the construction where we consider all possible sequences of $n$-covers instead of all possible $\omega$-covers.

\section{The Menger and Hurewicz conjectures}

Some of the classes can be treated without any special hypotheses.

Lemma 8. Assume that $\mathcal{J} \subseteq P(\mathbb{R})$ is closed under taking subsets and continuous images, and assume that $[0,1] \in \mathcal{J}$. Then $\mathcal{J}=P(\mathbb{R})$.

Proof. The subset $(0,1)$ of $[0,1]$ belongs to $\mathcal{J}$ and can be mapped continuously onto $\mathbb{R}$. Thus, every subset $X$ of $\mathbb{R}$ is a continuous image of some subset of $(0,1)$.

By the definition, every $\sigma$-compact set of reals satisfies $\mathrm{U}_{\text {fin }}(\Gamma, \Gamma)$. Moreover, every $\sigma$-compact set satisfies $\mathrm{S}_{\text {fin }}(\Omega, \Omega)$ [11. Since all of the properties in Figure 1 are closed under taking continuous images and $\mathrm{U}_{\text {fin }}(\Gamma, \mathcal{O}) \neq P(\mathbb{R})$, we have the following.

Corollary 9. None of the classes in Figure 1 which contain $\bigcup_{f i n}(\Gamma, \Gamma)$ or $\mathrm{S}_{f i n}(\Omega, \Omega)$ is hereditary.

A natural question is whether all nonhereditary sets in these classes are $\sigma$ compact. Related questions were raised by Menger and Hurewicz. Menger [13] conjectured that each set of reals satisfying $\mathrm{U}_{\text {fin }}(\Gamma, \mathcal{O})$ is $\sigma$-compact. Hurewicz [10] had a weaker conjecture that $\mathrm{U}_{\text {fin }}(\Gamma, \Gamma)$ implies $\sigma$-compactness. A Sierpinski set is a counter-example to both conjectures [11, but it was only recently [7, 11] that these conjecture were disproved in ZFC. Both refutations use a dichotomic argument: For an appropriate cardinal $\kappa \geq \aleph_{1}$, two independent examples are given for the case $\kappa=\aleph_{1}$ and for the case $\kappa>\aleph_{1}$. Our goal in the sequel is to explore the properties of two axiomatic-independent counter-examples to these conjectures.

We adopt the following setting from $[2$. Let $\overline{\mathbb{N}}=\mathbb{N} \cup\{\infty\}$ be the one point compactification of $\mathbb{N}$. A subset $A \subseteq \overline{\mathbb{N}}$ is open if $A \subseteq \mathbb{N}$, or $\infty \in A$ and $A$ is cofinite. Thus, if $A$ is a compact subset of $\overline{\mathbb{N}}$ and $\infty \notin A$, then $A$ is finite.

Let $\mathcal{Z} \subseteq{ }^{\mathbb{N}} \overline{\mathbb{N}}$ consist of the nondecreasing functions $f$ such that for each $n$ with $f(n)<\infty, f(n)<f(n+1)$. $\mathcal{Z}$ is a zero-dimensional metrizable compact space without isolated points, thus by a classical theorem of Brouwer it is homeomorphic to the Cantor space ${ }^{\mathbb{N}}\{0,1\}$. For each increasing finite sequence $s$ of natural numbers, let $q_{s} \in \mathcal{Z}$ be defined as

$$
q_{s}(k)= \begin{cases}s(k) & \text { if } k<|s|, \\ \infty & \text { otherwise }\end{cases}
$$

for each $k \in \mathbb{N}$. The set $Q$ of all these elements $q_{s}$ is dense in $\mathcal{Z}$. 
The following example is only a minor modification of the one given in [2], but the properties derived here for this example are stronger.

Theorem 10. There exists a (non- $\sigma$-compact) subset $H$ of $\mathcal{Z}$, such that:

(1) $|H|=\mathfrak{b}$,

(2) $H$ satisfies $\mathrm{S}_{1}(\Gamma, \mathcal{O})$ and all finite powers of $H$ satisfy $\mathrm{U}_{\text {fin }}(\Gamma, \Gamma)$, but $H \backslash Q$ does not satisfy $\mathrm{U}_{\text {fin }}(\Gamma, \Gamma)$, and

(3) If $\mathfrak{b}=\mathfrak{d}$, then $H \backslash Q$ does not satisfy $\bigcup_{\text {fin }}(\Gamma, \mathcal{O})$.

Proof. First, observe that an uncountable $\sigma$-compact set of reals contains a perfect set, and this is ruled out by the property $\mathrm{S}_{1}(\Gamma, \mathcal{O})$ [1]. The rest of the proof is divided into several lemmas. We take $H=B \cup Q$, where $B$ is the set described in the following lemma.

Lemma 11. There exists an unbounded subset $B=\left\{g_{\alpha}: \alpha<\mathfrak{b}\right\}$ of ${ }^{\mathbb{N}}$ where: Each $g_{\alpha}$ is increasing, $g_{\alpha} \leq^{*} g_{\beta}$ for each $\alpha<\beta$, and if $\mathfrak{b}=\mathfrak{d}$, then $B$ is dominating.

Proof. Start with $\left\{f_{\alpha}: \alpha<\mathfrak{b}\right\}$ unbounded and $\left\{h_{\alpha}: \alpha<\mathfrak{d}\right\}$ dominating, and by induction on $\alpha$, choose $g \in{ }^{\mathbb{N}} \mathbb{N}$ bounding $\left\{g_{\beta}: \beta<\alpha\right\}$, and if possible choose also $h \in{ }^{\mathbb{N}} \mathbb{N}$ bounding $\left\{h_{\beta}: \beta<\alpha\right\}$ (otherwise take $h \equiv 0$ ). Let $g_{\alpha}$ be an increasing function such that $g, h, f_{\alpha} \leq^{*} g_{\alpha}$.

According to a theorem of Hurewicz (see Recław [17]), a zero-dimensional set of reals $X$ satisfies $\mathrm{U}_{f i n}(\Gamma, \Gamma)$ (respectively, $\mathrm{U}_{f i n}(\Gamma, \mathcal{O})$ ) if, and only if, each continuous image of $X$ in ${ }^{\mathbb{N}} \mathbb{N}$ is bounded (respectively, not dominating). As $H \backslash Q=B$ is unbounded, it does not satisfy $\mathrm{U}_{\text {fin }}(\Gamma, \Gamma)$. If $\mathfrak{b}=\mathfrak{d}$, then $B$ is dominating, thus $H \backslash Q$ does not satisfy $\mathrm{U}_{f i n}(\Gamma, \mathcal{O})$.

We now show that $H$ satisfies $\mathrm{S}_{1}(\Gamma, \mathcal{O})$. A subset $A$ of ${ }^{\mathbb{N}} \mathbb{N}$ is strongly unbounded if for each $f \in{ }^{\mathbb{N}} \mathbb{N},\left|\left\{g \in A: g \leq^{*} f\right\}\right|<|A|$. Observe that $B$ is strongly unbounded. For a cardinal $\kappa$, a set of reals $X$ is $\kappa$-concentrated on a set $Y$ if for each open set $U \supseteq Y,|A \backslash U|<\kappa$.

Lemma 12. Assume that $A$ is a strongly unbounded subset of ${ }^{\mathbb{N}} \mathbb{N}$ and $\kappa=|A|$. Then:

(1) A is $\kappa$-concentrated on $Q$, and

(2) for each family $\mathscr{A}$ of open covers of $A \cup Q$, if $\kappa \leq \operatorname{non}\left(\mathrm{S}_{1}(\mathscr{A}, \mathcal{O})\right)$, then $A \cup Q$ satisfies $\mathrm{S}_{1}(\mathscr{A}, \mathcal{O})$.

Proof. (1) Assume that $U \supseteq Q$ is open. Then $\mathcal{Z} \backslash U$ is a closed and therefore compact subset of $\mathcal{Z}$. Since $\mathcal{Z} \backslash U$ is disjoint from $Q$, it is a compact (and therefore bounded) subset of ${ }^{\mathbb{N}} \mathbb{N}$. As $A$ is strongly unbounded, $|A \backslash U|=|A \cap(\mathcal{Z} \backslash U)|<\kappa$.

(2) Assume that $\mathcal{U}_{n} \in \mathscr{A}, n \in \mathbb{N}$. Enumerate $Q=\left\{q_{n}: n \in \mathbb{N}\right\}$, and choose for each $n U_{2 n} \in \mathcal{U}_{2 n}$ such that $q_{n} \in U_{2 n}$. Let $U=\bigcup_{n} U_{2 n}$. Then $|A \backslash U|<$ $\kappa \leq \operatorname{non}\left(\mathrm{S}_{1}(\mathscr{A}, \mathcal{O})\right)$, thus $A \backslash U$ satisfies $\mathrm{S}_{1}(\mathscr{A}, \mathcal{O})$. For each $n$ choose an element $U_{2 n+1} \in \mathcal{U}_{2 n+1}$ such that $A \backslash U \subseteq \bigcup_{n} U_{2 n+1}$. Then $\left\{U_{n}\right\}_{n \in \mathbb{N}}$ is a cover of $A \cup Q$.

We need the following extension of Lemma 2 of [2].

Lemma 13. Assume that $Q \subseteq X \subseteq \mathcal{Z}$ and $\Psi: X^{k} \rightarrow{ }^{\mathbb{N}} \mathbb{N}$ is continuous on $Q^{k}$. Then there exists $g \in{ }^{\mathbb{N}} \mathbb{N}$ such that for each $n$ and each $x_{1}, \ldots, x_{k} \in X$,

$$
\text { if } g(n)<\min \left\{x_{1}(n), \ldots, x_{k}(n)\right\} \text {, then } \Psi\left(x_{1}, \ldots, x_{k}\right)(n) \leq g(n) \text {. }
$$


Proof. For each $A \subseteq{ }^{\mathbb{N}} \overline{\mathbb{N}}$, let $A\lceil n=\{x\lceil n: x \in A\}$. $\mathcal{Z}\lceil n$ is a closed and therefore compact subset of $\overline{\mathbb{N}}^{n}$. For $\sigma \in \mathcal{Z} \backslash n$, write $q_{\sigma}$ for $q_{\sigma \uparrow m}$ where $m=1+$ $\max \{i<|\sigma|: \sigma(i)<\infty\}$. If $\sigma \in \mathcal{Z}\left\lceil n\right.$ and $I$ is a neighborhood of $q_{\sigma}$, then there exists a natural number $N$ such that for each $x \in \mathcal{Z}$ with $x\lceil n \in I\lceil n$ and $x(n)>N, x \in I$.

Fix $n$. For each $\vec{\sigma}=\left(\sigma_{1}, \ldots, \sigma_{k}\right) \in\left(\mathcal{Z}\lceil n)^{k}\right.$, choose a basic open neighborhood

$$
I_{\vec{\sigma}}=I_{\sigma_{1}} \times \ldots \times I_{\sigma_{k}} \subseteq \mathcal{Z}^{k}
$$

of $q_{\vec{\sigma}}=\left(q_{\sigma_{1}}, \ldots, q_{\sigma_{k}}\right)$ such that for each $\left(x_{1}, \ldots, x_{k}\right) \in X^{k} \cap I_{\vec{\sigma}}, \Psi\left(x_{1}, \ldots, x_{k}\right)(n)=$ $\Psi\left(q_{\vec{\sigma}}\right)(n)$ (this is possible because $\Psi$ is continuous on $Q^{k}$ ). For each $i=1, \ldots, k$, choose $N_{i}$ such that for all $x \in \mathcal{Z}$ with $x\left\lceil n \in I_{\sigma_{i}}\left\lceil n\right.\right.$ and $x(n)>N_{i}, x \in I_{\sigma_{i}}$. Define $N(\vec{\sigma}):=\max \left\{N_{1}, \ldots, N_{k}\right\}$. (So that if $\left(x_{1}\left\lceil n, \ldots, x_{k}\lceil n) \in I_{\sigma_{1}}\lceil n \times \ldots \times\right.\right.$ $I_{\sigma_{k}}\left\lceil n\right.$ and $x_{1}(n), \ldots, x_{k}(n)>N(\vec{\sigma})$, then $\left.\Psi\left(x_{1}, \ldots, x_{k}\right)(n)=\Psi\left(q_{\vec{\sigma}}\right)(n)\right)$.

The set $I_{\vec{\sigma}}^{(n)}=\left\{\left(x_{1}\left\lceil n, \ldots, x_{k}\lceil n):\left(x_{1}, \ldots, x_{k}\right) \in I_{\vec{\sigma}}\right\}\right.\right.$ is open in $\left(\mathcal{Z}\lceil n)^{k}\right.$ and the family $\left\{I_{\vec{\sigma}}^{(n)}: \vec{\sigma} \in\left(\mathcal{Z}\lceil n)^{k}\right\}\right.$ is a cover of $\left(\mathcal{Z}\lceil n)^{k}\right.$. By compactness it contains a finite subcover $\left\{I_{\vec{\sigma}_{1}}^{(n)}, \ldots, I_{\vec{\sigma}_{m}}^{(n)}\right\}$ of $\left(\mathcal{Z}\lceil n)^{k}\right.$. Take $N=\max \left\{N\left(\vec{\sigma}_{1}\right), \ldots, N\left(\vec{\sigma}_{m}\right)\right\}$, and define

$$
g(n)=\max \left\{N, \Psi\left(q_{\vec{\sigma}_{1}}\right)(n), \ldots, \Psi\left(q_{\vec{\sigma}_{m}}\right)(n)\right\} .
$$

For each $x_{1}, \ldots, x_{k} \in X$, let $i$ be such that $\left(x_{1}\left\lceil n, \ldots, x_{k}\lceil n) \in I_{\vec{\sigma}_{i}}^{(n)}\right.\right.$. If $x_{1}(n), \ldots$, $x_{k}(n)>N$, then $\Psi\left(x_{1}, \ldots, x_{k}\right)(n)=\Psi\left(q_{\vec{\sigma}_{i}}\right)(n) \leq g(n)$.

It remains to show that all finite powers of $H$ satisfy $\bigcup_{f i n}(\Gamma, \Gamma)$. We will show, by induction on $k$, that all continuous images in ${ }^{\mathbb{N}} \mathbb{N}$ of the finite powers $H^{k}$ of $H$ are bounded.

Assume that $\Psi: H^{k+1} \rightarrow{ }^{\mathbb{N}} \mathbb{N}$ is continuous. We may assume that all elements in the image of $\Psi$ are increasing. Let $g \in{ }^{\mathbb{N}} \mathbb{N}$ be (increasing, and) as in Lemma 13. By Lemma 11, there exists $\alpha<\mathfrak{b}$ such that the set $A=\left\{n: g(n)<g_{\alpha}(n)\right\}$ is infinite, and for each $\beta>\alpha, A \subseteq^{*}\left\{n: g(n)<g_{\beta}(n)\right\}$. Let $\left\{a_{n}\right\}_{n \in \mathbb{N}}$ be an increasing enumeration of $A$, and define $h \in{ }^{\mathbb{N}} \mathbb{N}$ by $h(n)=g\left(a_{n}\right)$.

By Lemma 13, for all $\alpha_{1}, \ldots, \alpha_{k}>\alpha$ and all but finitely many $n$,

$$
\Psi\left(g_{\alpha_{1}}, \ldots, g_{\alpha_{k}}\right)(n) \leq \Psi\left(g_{\alpha_{1}}, \ldots, g_{\alpha_{k}}\right)\left(a_{n}\right) \leq g\left(a_{n}\right)=h(n) .
$$

For each $f \in\left\{g_{\beta}: \beta \leq \alpha\right\} \cup Q$ and each $m=1, \ldots, k+1$ define $\Psi_{m, f}: H^{k} \rightarrow{ }^{\mathbb{N}}$ by

$$
\Psi_{m, f}\left(x_{1}, \ldots, x_{k}\right)=\Psi\left(x_{1}, \ldots, x_{m-1}, f, x_{m+1}, \ldots, x_{k}\right) .
$$

By the induction hypothesis, the image of each function $\Psi_{m, f}$ is bounded. As there are less than $\mathfrak{b}$ many such functions, we have that $\Psi\left[H^{k+1}\right]$ is bounded.

The proof that $H$ satisfies $\bigcup_{f i n}(\Gamma, \Gamma)$ is similar [2]. This completes the proof of Theorem 10 .

To state the following corollary, we need some preliminaries. Consider the collection $\Omega^{g p}$ of open $\omega$-covers $\mathcal{U}$ of $X$ such that there exists a partition $\mathcal{P}$ of $\mathcal{U}$ into finite sets such that for each finite $F \subseteq X$ and all but finitely many $\mathcal{F} \in \mathcal{P}$, there exists $U \in \mathcal{F}$ such that $F \subseteq U$. $\mathrm{S}_{f i n}\left(\Omega, \Omega^{g p}\right)$ is strictly stronger than $\mathrm{U}_{f i n}(\Gamma, \Gamma)$, and it is also strictly stronger than $\mathrm{S}_{\text {fin }}(\Omega, \Omega)$. A set $X \subseteq \mathbb{R}$ is meager additive if for each meager set $M \subseteq \mathbb{R}, X+M$ is meager. If $X$ satisfies $\mathrm{U}_{\text {fin }}(\Gamma, \Gamma)$ and has strong measure zero (both properties follow from $\mathrm{S}_{1}\left(\Omega, \Omega^{g p}\right)$ ), then it is meager-additive [16], but $\mathrm{S}_{1}\left(\Omega, \Omega^{g p}\right)$ is strictly stronger than being meager additive: Consider the 
set $X$ of Theorem 4, Then $X$ is meager additive [8, and therefore so is $X \backslash[\mathbb{N}]<\aleph_{0}$, but $X \backslash[\mathbb{N}]^{<\aleph_{0}}$ does not even satisfy $\mathrm{U}_{\text {fin }}(\Gamma, \mathcal{O})$. Let SMZ denote the collection of strong measure zero sets of reals. $\operatorname{cov}(\mathcal{M}) \leq \operatorname{non}(\mathrm{SMZ})$, and strict inequality is consistent. A set of reals $X$ is perfectly meager if for each perfect set $P, X \cap P$ is meager in the relative topology of $P$. It is universally meager if it does not contain an injective Borel image of a nonmeager set of reals.

Corollary 14. The set $H$ has the following properties:

(1) $\mathrm{S}_{\text {fin }}\left(\Omega, \Omega^{g p}\right)$ and $\mathrm{S}_{1}(\Gamma, \mathcal{O})$.

(2) It is universally meager (in particular, it is perfectly meager).

(3) If $\mathfrak{b} \leq \operatorname{non}(\mathrm{SMZ})$, then $H$ satisfies $\mathrm{S}_{1}\left(\Omega, \Omega^{g p}\right)$. In particular, in this case it is meager-additive.

Proof. (1) $X$ satisfies $\mathrm{S}_{f i n}\left(\Omega, \Omega^{g p}\right)$ if, and only if, all finite powers of $X$ satisfy $\mathrm{U}_{\text {fin }}(\Gamma, \Gamma)[12$.

(2) An uncountable set of reals $X$ satisfying $\mathrm{S}_{1}(\Gamma, \mathcal{O})$ cannot contain a perfect set of reals [1]. Zakrzewski [27, Proposition 2.3] proved that if $X$ satisfies $\cup_{f i n}(\Gamma, \Gamma)$ and does not contain a perfect set, then $X$ is universally meager.

(3) If $\mathfrak{b} \leq$ non $(\mathrm{SMZ})$, then $H$ is non(SMZ)-concentrated on the countable set $Q$, which implies that $H$ has strong measure zero. By [26], $\mathrm{S}_{f i n}\left(\Omega, \Omega^{g p}\right) \cap \mathrm{SMZ}=$ $\mathrm{S}_{1}\left(\Omega, \Omega^{g p}\right)$.

Corollary 14(3) extends Theorem 2(1) of [1], which asserts that if $\mathfrak{b}=\aleph_{1}$, then there exists a meager-additive set of reals. Corollary 14 (2) implies a negative answer to Steprans' Question 5 from [21]: Does the inequality non $(\mathcal{M})>\aleph_{1}$ imply that no set of size greater than $\aleph_{1}$ is perfectly meager? The answer is "No", since $H$ is universally meager, and $\mathfrak{b}>\aleph_{1}$ is consistent with the assumption of the question.

Problem 15. $\mathrm{S}_{1}(\Gamma, \Gamma)$ ?

(1) Does the set $H$ constructed in Theorem 10 satisfy

(2) Do all finite powers of $H$ satisfy $\mathrm{S}_{1}(\Gamma, \mathcal{O})$ ?

The methods of [19] may be relevant to Problem 15(1). A positive answer to Problem 15) (2) would imply that $H$ satisfies $S_{1}(\Gamma, \Omega)$.

We now treat Menger's Conjecture. A set of reals $X$ satisfies $S_{f i n}(\Omega, \Omega)$ if, and only if, all finite powers of $X$ satisfy $\mathrm{U}_{f i n}(\Gamma, \mathcal{O})$ [11. We do not know whether there exists in ZFC a (non- $\sigma$-compact) set of size $\mathfrak{d}$ satisfying $\mathrm{S}_{\text {fin }}(\Omega, \Omega$ ). It is known that $\operatorname{cov}(\mathcal{M})=\mathfrak{c}$ is enough to deduce the existence of such a set [11]. We will show that this can also be deduced from an assumption which contradicts $\operatorname{cov}(\mathcal{M})=\mathfrak{c}$. A subset $F$ of ${ }^{\mathbb{N}} \mathbb{N}$ is finitely dominating if for each $g \in{ }^{\mathbb{N}}$ there exist $k$ and $f_{1}, \ldots, f_{k} \in{ }^{\mathbb{N}} \mathbb{N}$ such that $g(n) \leq{ }^{*} \max \left\{f_{1}(n), \ldots, f_{k}(n)\right\}$. Let $\mathfrak{D}_{\text {fin }}$ denote the collection of sets $F$ of increasing elements of ${ }^{\mathbb{N}} \mathbb{N}$, which are not finitelydominating, and let add $\left(\mathfrak{D}_{\text {fin }}\right)=\min \left\{|\mathcal{F}|: \mathcal{F} \subseteq \mathfrak{D}_{\text {fin }}\right.$ and $\left.\cup \mathcal{F} \notin \mathfrak{D}_{\text {fin }}\right\}$. If $\operatorname{cov}(\mathcal{M})=$ $\mathfrak{c}$, then $\operatorname{add}\left(\mathfrak{D}_{\text {fin }}\right)=2$, but $\mathrm{NCF}$ is equivalent to $\operatorname{add}\left(\mathfrak{D}_{\text {fin }}\right)>2$, and if $\mathfrak{u}<\mathfrak{g}$, then $\operatorname{add}\left(\mathfrak{D}_{\text {fin }}\right)=\mathfrak{c}$ [3, full version].

Theorem 16. There exists a (non- $\sigma$-compact) subset $M$ of $\mathcal{Z}$, such that:

(1) $|M|=\mathfrak{d}$,

(2) $M$ satisfies $\mathrm{S}_{1}(\Gamma, \mathcal{O})$, but $M \backslash Q$ does not satisfy $\mathrm{U}_{\text {fin }}(\Gamma, \mathcal{O})$,

(3) if $N C F$ holds, then $M$ satisfies $\mathrm{U}_{\text {fin }}(\Gamma, \Omega)$; and

(4) if $\mathfrak{b}=\mathfrak{d} 1$ or $\operatorname{add}\left(\mathfrak{D}_{\text {fin }}\right)=\mathfrak{d}$, then $M$ satisfies $\mathrm{S}_{\text {fin }}(\Omega, \Omega)$.

\footnotetext{
${ }^{1}$ Equivalently, a union of less than $\mathfrak{d}$ nondominating subsets of ${ }^{\mathbb{N}} \mathbb{N}$ is not dominating.
} 
Proof. The proof is similar to that of Theorem 10. We describe only the differences. Here, we take $M=D \cup Q$, where $D$ is defined in the following lemma.

Lemma 17. There exists a dominating subset $D=\left\{g_{\alpha}: \alpha<\mathfrak{d}\right\}$ of ${ }^{\mathbb{N}} \mathbb{N}$ where each $g_{\alpha}$ is increasing, and for each $f \in \mathbb{N}_{\mathbb{N}}$ there exists $\alpha_{0}<\mathfrak{d}$ such that for any finite set $F \subseteq \mathfrak{d} \backslash \alpha_{0}, f(n)<\min \left\{g_{\beta}(n): \beta \in F\right\}$ for infinitely many $n$.

Proof. Let $\left\{f_{\alpha}: \alpha<\mathfrak{d}\right\}$ be a dominating subset of ${ }^{\mathbb{N}} \mathbb{N}$. We construct $g_{\alpha}$ by induction on $\alpha<\mathfrak{d}$. Assume that $g_{\beta}$ are constructed for $\beta<\alpha$, such that for each $\beta<\alpha$ and each finite set $F \subseteq \alpha \backslash \beta$, the set

$$
X_{\beta, F}=\left\{n: f_{\beta}(n)<\min \left\{g_{\gamma}(n): \gamma \in F\right\}\right\}
$$

is infinite. For each $\beta<\alpha$ and finite $F \subseteq \alpha \backslash \beta$, let $h_{\beta, F} \in{ }^{\mathbb{N}}$ be the increasing enumeration of $X_{\beta, F}$. The collection

$$
\left\{f_{\beta} \circ h_{\beta, F}: \beta<\alpha, F \text { a finite subset of } \alpha \backslash \beta\right\}
$$

has less than $\mathfrak{d}$ many elements, thus there exists an increasing $g_{\alpha} \in{ }^{\mathbb{N}} \mathbb{N}$ such that $f_{\alpha} \leq^{*} g_{\alpha}$, and for each $\beta$ and $F, g_{\alpha} \not^{*} f_{\beta} \circ h_{\beta, F}$. Therefore $g_{\alpha} \circ h_{\beta, F} \not^{*} f_{\beta} \circ h_{\beta, F}$, thus

$$
f_{\beta}(n)<\min \left\{g_{\gamma}(n): \gamma \in F \cup\{\alpha\}\right\}
$$

for infinitely many $n$. This completes the inductive step.

We need to prove that if $\operatorname{add}\left(\mathfrak{D}_{\text {fin }}\right)>2$, then $M$ satisfies $\mathrm{U}_{\text {fin }}(\Omega, \Omega)$, and if $\mathfrak{b}=\mathfrak{d}$ or $\operatorname{add}\left(\mathfrak{D}_{\text {fin }}\right)=\mathfrak{d}$, then $M$ satisfies $\mathrm{S}_{\text {fin }}(\Omega, \Omega)$. The proof of the first assertion uses arguments similar to the following ones, and we omit it.

Assume that $\operatorname{add}\left(\mathfrak{D}_{\text {fin }}\right)=\mathfrak{d}$ (respectively, $\mathfrak{b}=\mathfrak{d}$ ). Assume that $M^{k}$ satisfies $\mathrm{U}_{\text {fin }}(\Gamma, \Omega)$ (respectively, $\mathrm{U}_{\text {fin }}(\Gamma, \mathcal{O})$ ). Assume that $\Psi: M^{k+1} \rightarrow{ }^{\mathbb{N}} \mathbb{N}$ is continuous and all elements in its image are increasing. By [23] (respectively, by Hurewicz' Theorem), it suffices to show that $\Psi\left[M^{k+1}\right]$ is not finitely dominating (respectively, not dominating).

Let $g \in \mathbb{N}^{\mathbb{N}}$ be increasing and as in Lemma 13, and take $\alpha_{0}<\mathfrak{d}$ as in Lemma 17 Assume that $F=\left\{g_{j}^{i}: i<m, j \leq k\right\} \subseteq\left\{g_{\alpha}: \alpha \in \mathfrak{d} \backslash \alpha_{0}\right\}$. Then there exists an infinite set $A \subseteq \mathbb{N}$ such that $g(n)<\min \left\{g_{j}^{i}(n): i<m, j \leq k\right\}$ for each $n \in A$, and if $y_{i}=\Psi\left(g_{0}^{i}, \ldots, g_{k}^{i}\right)$ for each $i<m$, then by Lemma 13 ,

$$
\max \left\{y_{0}(n), \ldots, y_{m-1}(n)\right\} \leq g(n)
$$

for each $n \in A$. Thus, $\Psi\left[\left\{g_{\alpha}: \alpha \in \mathfrak{d} \backslash \alpha_{0}\right\}^{k+1}\right]$ is not finitely dominating. It follows that $\Psi\left[M^{k+1}\right]$ is a union of less than $\mathfrak{d}$ many sets which are not finitely dominating.

Remark 18. If one only wants to obtain (1) and (2) of Theorem 16, then it suffices to take $M=\Psi[D] \cup \mathbb{Q}$, where $D$ is any strongly unbounded subset of ${ }^{\mathbb{N}} \mathbb{N}$ and $\Psi:{ }^{\mathbb{N}} \rightarrow \mathbb{R} \backslash \mathbb{Q}$ is a homeomorphism. Essentially, this idea goes back to Rothberger.

\section{ACKNOWLEDGEMENTS}

We thank Roman Pol, Taras Banakh, and Lubomyr Zdomsky for making crucial comments on earlier versions of this paper, and the referee for extending and simplifying our proof of Proposition 2. We also thank Tomasz Weiss for fruitful discussions.

Chaber and Pol proposed another approach to the Menger and Hurewicz conjectures; see [5]. 


\section{REFERENCES}

[1] T. Bartoszyński, Remarks on small sets of reals, Proceedings of the American Mathematical Society 131, 625-630. MR1933355(2003i:03052)

[2] T. Bartoszyński and S. Shelah, Continuous images of sets of reals, Topology and its Applications 116 (2001), 243-253. MR:1855966 (2002f:03090)

[3] T. Bartoszyński, S. Shelah, and B. Tsaban, Additivity properties of topological diagonalizations, The Journal of Symbolic Logic 68 (2003), 1254-1260. (Full version: math.LO/0112262) MR.2017353 (2004k:03094)

[4] A. R. Blass, Combinatorial cardinal characteristics of the continuum, in: Handbook of Set Theory (M. Foreman, A. Kanamori, and M. Magidor, eds.), Kluwer Academic Publishers, Dordrecht, to appear.

[5] J. Chaber and R. Pol, A remark on Fremlin-Miller theorem concerning the Menger property and Michael concentrated sets, preprint.

[6] E. K. van Douwen, The integers and topology, in: Handbook of Set Theoretic Topology (K. Kunen and J. Vaughan, eds.), North-Holland, Amsterdam, 1984, 111-167. MR0776622 (87f:54008)

[7] D. H. Fremlin and A.W. Miller, On some properties of Hurewicz, Menger and Rothberger, Fundamenta Mathematica 129 (1988), 17-33. MR0954892 (89g:54061)

[8] F. Galvin and A.W. Miller, $\gamma$-sets and other singular sets of real numbers, Topology and it Applications 17 (1984), 145-155. MR0738943 (85f:54011)

[9] J. Gerlits and Zs. Nagy, Some properties of $C(X)$, I, Topology and its Applications 14 (1982), 151-161. MR0667661 (84f:54021)

[10] W. Hurewicz, Über eine Verallgemeinerung des Borelschen Theorems, Mathematische Zeitschrift 24 (1925), 401-421.

[11] W. Just, A. W. Miller, M. Scheepers, and P. Szeptycki, Combinatorics of open covers II, Topology and Its Applications, 73 (1996), 241-266. MR.1419798 (98g:03115a)

[12] Lj. D. R. Kočinac and M. Scheepers, Combinatorics of open covers (VII): Groupability, Fundamenta Mathematicae 179 (2003), 131-155. MR2029229 (2005c:54020)

[13] M. K. Menger, Einige Überdeckungssätze der Punktmengenlehre, Sitzungsberichte der Wiener Akademie 133 (1924), 421-444.

[14] A. W. Miller, On the length of Borel hierarchies, Annals of Mathematical Logic 16 (1979), 233-267. MR0548475 (80m:04003)

[15] A. W. Miller, A Nonhereditary Borel-cover $\gamma$-set, Real Analysis Exchange 29 (2003/4), 601-606. MR2083799

[16] A. Nowik, M. Scheepers, and T. Weiss, The algebraic sum of sets of real numbers with strong measure zero sets, J. Symbolic Logic 63 (1998), 301-324. MR1610427 (99c:54049)

[17] I. Recław, Every Luzin set is undetermined in point-open game, Fundamenta Mathematicae 144 (1994), 43-54. MR:1271477 (95f:04005)

[18] M. Scheepers, Combinatorics of open covers I: Ramsey theory, Topology and its Applications 69 (1996), 31-62. MR1378387 (97h:90123)

[19] M. Scheepers, Sequential convergence in $\mathrm{C}_{p}(X)$ and a covering property, East-West Journal of Mathematics 1 (1999), 207-214. MR1727383 (2000i:54014)

[20] M. Scheepers and B. Tsaban, The combinatorics of Borel covers, Topology and its Applications 121 (2002), 357-382. MR.1908999 (2003e:03091)

[21] J. Steprans, Question about which I have thought, preprint: http://www.math. yorku.ca/Who/Faculty/Steprans/Research/q.pdf

[22] B. Tsaban, A topological interpretation of $\mathfrak{t}$, Real Analysis Exchange 25 (1999/2000), 391404. MR.1691758 (2000j:03066)

[23] B. Tsaban, A diagonalization property between Hurewicz and Menger, Real Analysis Exchange 27 (2001/2002), 757-763. MR.1923165 (2004c:26001)

[24] B. Tsaban, Selection principles and the minimal tower problem, Note di Matematica 22 (2003), 53-81.

[25] B. Tsaban, Strong $\gamma$-sets and other singular spaces, Topology and its Applications, to appear. 
[26] B. Tsaban and T. Weiss, Products of special sets of real numbers, Real Analysis Exchange, to appear.

[27] P. Zakrzewski, Universally meager sets, Proceedings of the American Mathematical Society 129 (2000), 1793-1798. MR1814112 (2001m:03097)

Department of Mathematics and Computer Science, Boise State University, Boise, IDAHO 83725

E-mail address: tomek@math.boisestate.edu

$U R L:$ http://math. boisestate.edu/ ${ }^{\sim}$ tomek

Department of Mathematics and Computer Science, Bar-Ilan University, Ramat-Gan 52900, ISRAEL

E-mail address: tsaban@macs.biu.ac.il

URL: http://www.cs.biu.ac.il/ tsaban

Current address: Department of Applied Mathematics and Computer Science, The Weizmann Institute of Science, Rehovot 76100, Israel

E-mail address: boaz.tsaban@weizmann.ac.il 